\title{
Decentralized Multi-Agent Advantage Actor-Critic
}

This paper was downloaded from TechRxiv (https://www.techrxiv.org).

\section{LICENSE}

CC BY 4.0

SUBMISSION DATE / POSTED DATE

14-02-2022 / 22-02-2022

CITATION

Barnes, Scott (2022): Decentralized Multi-Agent Advantage Actor-Critic. TechRxiv. Preprint. https://doi.org/10.36227/techrxiv.19166384.v1

$\mathrm{DOI}$ 


\title{
Decentralized Multi-Agent Advantage Actor-Critic
}

\author{
Scott G. Barnes \\ Department of Mechanical \\ \& Aerospace Engineering \\ The George Washington University \\ scottgbarnes@gwu.edu
}

\author{
Dustin J. Webb \\ School of Computing \\ University of Utah \\ dustin@cs.utah.edu
}

\author{
Adam M. Wickenheiser \\ Department of Mechanical \\ \& Aerospace Engineering \\ The George Washington University \\ amwick@gwu .edu
}

\begin{abstract}
We present a decentralized advantage actor-critic algorithm that utilizes learning agents in parallel environments with synchronous gradient descent. This approach decorrelates agents' experiences, stabilizing observations and eliminating the need for a replay buffer, requires no knowledge of the other agents' internal state during training or execution, and runs on a single multi-core CPU.
\end{abstract}

\section{Introduction}

Deep reinforcement learning (RL) has demonstrated success in a range of challenging fields, including video games [11], robotic control [3], and natural language processing [1]. However, the focus of most deep RL research has been on training individual actors in fixed environments. As multi-agent environments are inherently nonstationary, these methods are poorly suited for this application.

The primary challenge with multi-agent $\mathrm{RL}$ is that each agent's policy changes during training. As a result, the observations of individual agents are not stationary through time, preventing the use of traditional experience replay. This introduces stability and convergence issues with Q-learning techniques, which rely heavily on replay buffers. Similarly, policy gradient methods exhibit high variance in multi-agent environments. Model-based optimization techniques have demonstrated success in simple multi-agent environments but require a differentiable model of the environment and stringent assumptions on the agents' interactions, making them impractical for many tasks [7].

Decentralized Multi-Agent Advantage Actor-Critic (DMAAC) is a multi-agent learning algorithm that is decentralized in training and execution (each agent uses only local observations), agnostic to the structure of the environment and the policies of other agents, and stably converges to successful policies without the use of experience replay. It utilizes multiple learning agents in parallel environments and accumulated gradients with synchronous updates to decorrelate sequential agent experiences and stabilize training in dynamic environments. DMAAC is lightweight and non-distributed: it runs on a single multi-core CPU and trains nearly an order of magnitude faster than multi-agent RL algorithms that exhibit comparable performance.

DMAAC is an extension of Multi-Agent Deep Deterministic Policy Gradients (MADDPG) [7] and Asynchronous Advantage Actor-Critic (A3C) [10]. A3C demonstrated the power of parallel actorlearners through state-of-the-art performance in single-agent domains [10]. Similarly, MADDPG demonstrated superior performance to established algorithms in multi-agent environments [7]. Both methods are discussed at length in Section 2 . 


\section{Related Work}

Established reinforcement learning algorithms, such as Q-learning and policy gradients, have been shown to perform poorly in multi-agent environments. Q-learning methods suffer from exploration and convergence issues due to other agents' policies changing during training, which makes the environment non-stationary [9], while policy gradients exhibit high variance in multi-agent domains [7]. Some of these issues have been addressed through the use of distributed learning architectures [2], sharing of correlated Q-values with other agents [5], and the use of heuristic guided policy search [12]. Even with these modifications, the performance of these algorithms leaves significant room for improvement.

Recently, MADDPG demonstrated state-of-the-art performance in multi-agent environments. It uses a centralized critic during training, which observes actions of all agents to account for changes in their policies over time, making observations stationary. This allows for the use of a replay buffer, which decorrelates observations and stabilizes training. During execution, each model only uses local observations. MADDPG outperformed DDPG, REINFORCE, Actor-Critic, TRPO, and DQN in multiple cooperative and competitive environments [7].

Our approach differs significantly from MADDPG. It does not use a shared critic or a replay buffer; instead, multiple environments are executed in parallel and gradients are aggregated in batches. The use of parallel-actor learners has been shown to decorrelate sequential agent experiences [10].

The first deep RL algorithm to utilize parallel actor-learners was a variant of Sarsa, which performed peer-to-peer sharing of large gradients to expedite training [4]. However, this area remained largely unexplored until the release of A3C. A3C utilizes parallel actor-learners with asynchronous updates from accumulated gradients across $N$ environments to learn actor policies in single-agent environments, demonstrating state-of-the-art performance in Atari games [10].

DMAAC is a variant of A3C; however, there are significant differences between the two. First, DMAAC uses $M * N$ local models with $M$ global models for $M$ agents in $N$ environments. It also use synchronous, batched updates, which ensures that the agents' policies remain unchanged and are consistent across all environments between updates. This makes each batch of observations stationary, stabilizing training.

\section{Background}

\subsection{Actor-Critic Methods}

Actor-only methods, such as Q-learning, use simulation to directly sample state-action-reward experiences from a cost function to update model parameters via gradient descent in the direction of error minimization [8]. In contrast, critic-only methods approximate a value function over a set of states, which can then be used to calculate a near-optimal policy. However, actor-only methods exhibit high variance in gradient estimations, while the policies of critic-only methods tend to be unreliable despite near-optimal value approximations. Actor-critic techniques combine the strengths of these methods by updating an actor's policy parameters with the learned value function approximation during training [6].

We use the policy and value gradients ( $d \theta_{p}$ and $d \theta_{v}$, respectively) defined in [10]. The policy advantage, $\alpha$, measures the difference between the model's prediction and the actual value of a state. This encourages exploration of states in which the model's prediction is poor. The policy entropy $H(\pi)$ measures the distribution of action probabilities, increasing exploration of policies in which the action probability variance is large. $\beta$ is a constant that defines the entropy's weight. In these terms, the updates to the policy and value gradients are given by:

$$
\begin{gathered}
d \theta_{v}=d \theta_{v}+\partial\left(R-V\left(s_{t}, \theta_{v}\right)\right)^{2} / \partial \theta_{v} \\
d \theta_{p}=d \theta_{p}+\alpha \nabla_{\theta_{p}} \log \pi\left(a_{t} \mid s_{t}, \theta_{p}\right)+\beta H(\pi) \\
\alpha=R-V\left(s_{t}, \theta_{v}\right)
\end{gathered}
$$




\subsection{Multi-Agent Reinforcement Learning}

The fundamental challenge with multi-agent reinforcement learning is that the policy of each agent is updated independently during training. As a result, the distribution of outcomes $s^{\prime}$ differs for unique policies $\pi$ and $\pi^{\prime}$ over $N$ updates for a given set of state-action pairs:

$$
P\left(s^{\prime} \mid s, a, \pi_{1}, \ldots \pi_{N}\right) \neq P\left(s^{\prime} \mid s, a, \pi_{1}^{\prime}, \ldots \pi_{N}^{\prime}\right)
$$

This invalidates the Markov assumption and precludes the use of a traditional replay buffer, as the policies of other agents are non-stationary from the viewpoint of any single agent [7]. This violates necessary conditions for convergence. MADDPG addresses this issue by learning a shared critic that models the policies of all agents. This makes observations stationary, as the models are explicitly conditioned on the actions of other agents [7]. However, this assumes that the actions of other agents are observable during training.

\section{Method}

\subsection{DMAAC Algorithm}

DMAAC uses a fundamentally different method of stabilizing training in multiagent environments. Rather than use a replay buffer, we run $N$ environments in parallel, each with $M$ agents and randomly generated initial conditions (from the same distribution), to sample from a broad set of observations simultaneously. As demonstrated in [10], this decorrelates sequential observations and eliminates the need for a replay buffer.

Additionally, DMAAC uses synchronous, batched gradient updates. Actor-learners accumulate gradients during simulation, but they are not applied to the local models. Instead, they are applied simultaneously to global models at fixed intervals. This means that each batch of gradients is sampled from set of observations over which the policies of each agent is fixed. As a result, the Markov assumptions are not violated and the environment is stationary. This eliminates the need for a shared critic, as stationary updates do not need to be ex-

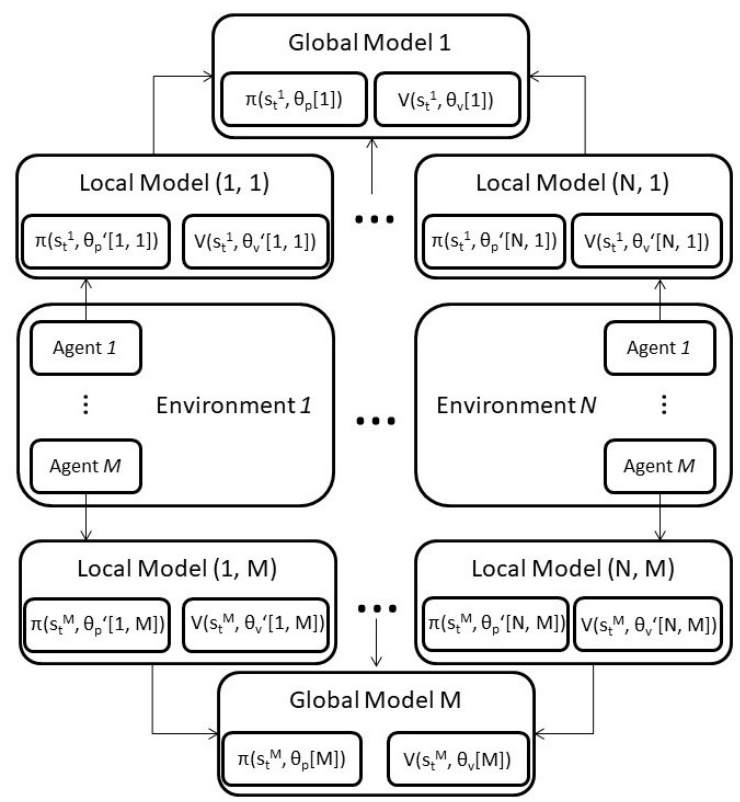

Figure 1: DMAAC Training Architecture plicitly conditioned on the actions of other agents.

DMAAC is fully decentralized in training and execution, does not use a shared critic, and does not require explicit knowledge of the other agents' policies or actions. Each agent's model only uses local observations and learns its own actor and critic. We demonstrate that if the external state of other agents is available at least intermittently, models trained with DMAAC can learn a successful collaborative policy.

Training is performed with $M *(N+1)$ models. $M * N$ local models accumulate gradients in parallel for $T$ time steps. These gradients are then used to update $M$ global models at each interval of $T$. The local models are synchronized with the parameters of the updated global models, and the process is repeated until convergence. In execution, the global models are applied to each agent.

Pseudo-code for the entire DMAAC algorithm is provided in Appendix $\mathrm{A}$ 


\section{Conclusion}

We have introduced a decentralized multi-agent actor-critic algorithm that does not require the use of a replay buffer, shared critic, or a dynamic model of the environment. Future work will include applying DMAAC to competitive environments and exploring methods of utilizing methods of both DMAAC and MADDPG to improve performance, such as the use of a centralized critic and bootstrapping early DMAAC training with MADDPG for faster convergence.

\section{Appendix A DMAAC Algorithm}

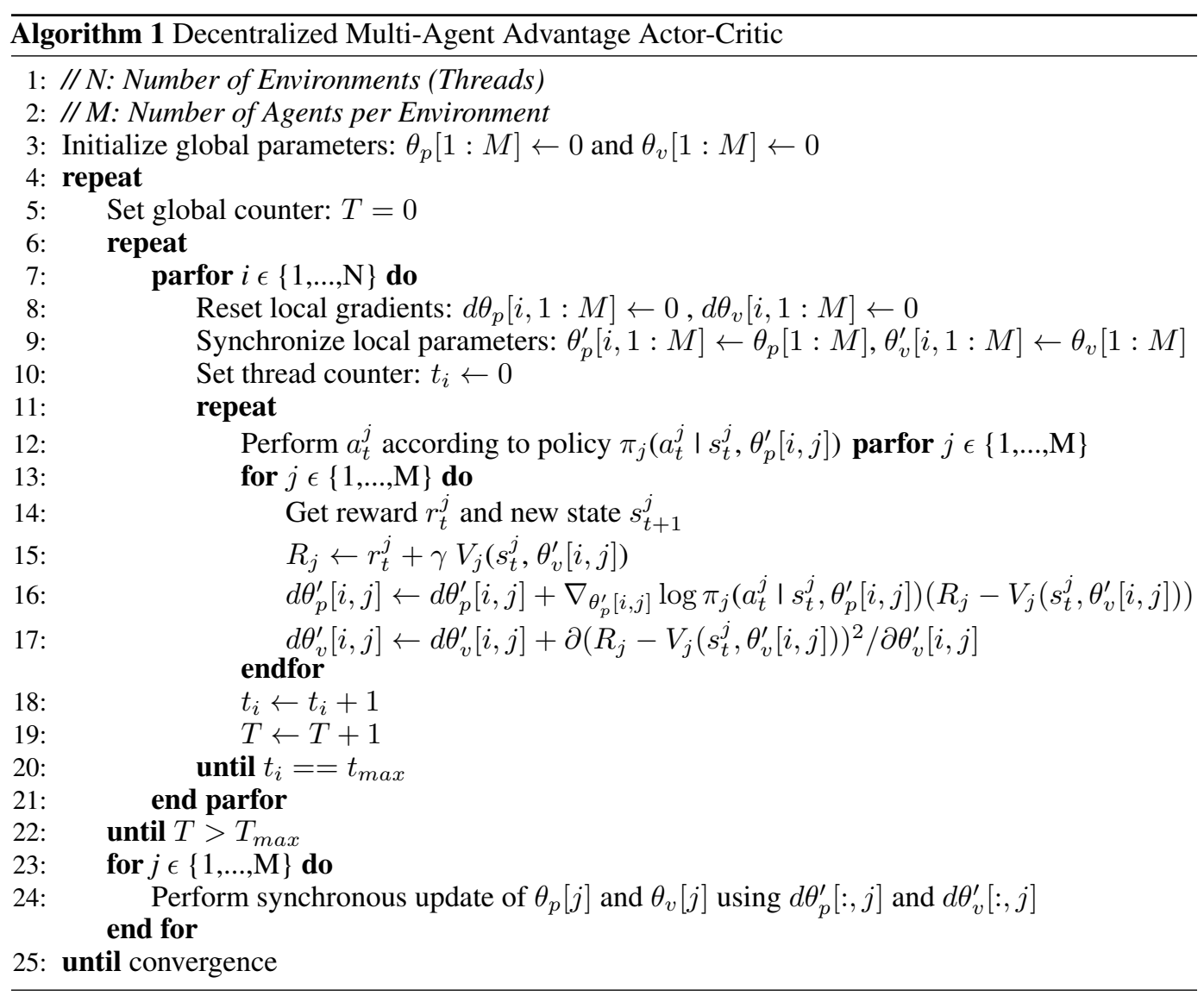

\section{References}

[1] K. Arulkumaran, M. P. Deisenroth, M. Brundage, and A. A. Bharath. A brief survey of deep reinforcement learning. CoRR, 1708.05866, 2017.

[2] C. Cheng, Z. Zhu, B. Xin, and C. Chen. A multi-agent reinforcement learning algorithm based on stackelberg game. In 2017 6th Data Driven Control and Learning Systems (DDCLS), pages 727-732, May 2017.

[3] I. Grondman, L. Busoniu, G. A. D. Lopes, and R. Babuska. A survey of actor-critic reinforcement learning: Standard and natural policy gradients. IEEE Transactions on Systems, Man, and Cybernetics, Part C (Applications and Reviews), 42(6):1291-1307, Nov 2012.

[4] M. J. Grounds and D. Kudenko. Parallel reinforcement learning with linear function approximation. In AAMAS, 2007.

[5] H. Guo and Y. Meng. Distributed reinforcement learning for coordinate multi-robot foraging. Journal of Intelligent and Robotic Systems, 60(3-4):531, 2010. 
[6] V. R. Konda and J. N. Tsitsiklis. Actor-critic algorithms. In S. A. Solla, T. K. Leen, and K. Müller, editors, Advances in Neural Information Processing Systems 12, pages 1008-1014. MIT Press, 2000.

[7] R. Lowe, Y. Wu, A. Tamar, J. Harb, P. Abbeel, and I. Mordatch. Multi-agent actor-critic for mixed cooperative-competitive environments. CoRR, abs/1706.02275, 2017.

[8] P. Marbach and J. N. Tsitsiklis. Simulation-based optimization of markov reward processes. IEEE Transactions on Automatic Control, 46(2):191-209, Feb 2001.

[9] L. Matignon, G. J. Laurent, and N. Le Fort-Piat. Independent reinforcement learners in cooperative markov games: a survey regarding coordination problems. The Knowledge Engineering Review, 27(1):1-31, 2012.

[10] V. Mnih, A. P. Badia, M. Mirza, A. Graves, T. P. Lillicrap, T. Harley, D. Silver, and K. Kavukcuoglu. Asynchronous methods for deep reinforcement learning. CoRR, abs/1602.01783, 2016.

[11] V. Mnih, K. Kavukcuoglu, D. Silver, A. Graves, I. Antonoglou, D. Wierstra, and M. A. Riedmiller. Playing atari with deep reinforcement learning. CoRR, 1312.5602, 2013.

[12] W. Zemzem and M. Tagina. Cooperative multi-agent reinforcement learning in a large stationary environment. In 2017 IEEE/ACIS 16th International Conference on Computer and Information Science (ICIS), pages 365-371, May 2017. 